\title{
AUTOMATED CORPUS CALLOSUM SEGMENTATION IN MIDSAGITTAL BRAIN MR IMAGES
}

\author{
Yue Li $^{1}$, Huiquan Wang ${ }^{2}$, Nizam Ahmed ${ }^{3}$ and Mrinal Mandal ${ }^{4}$ \\ ${ }^{1,2,4}$ Department of Electrical and Computer Engineering, University of Alberta, Canada \\ ${ }^{3}$ Department of Medicine, University of Alberta, Canada
}

\begin{abstract}
Corpus Callosum (CC) is an important white-matter structure in the human brain. Magnetic resonance imaging (MRI) is a non-invasive medical imaging technique that provides high resolution images for the structures. Segmentation is an important step in medical image analysis. This paper proposes a fully automated technique for segmentation of CC on the midsagittal slice of T1-weighted brain MR images. The proposed technique consists of three modules. First it clusters all homogenous regions in the image with an adaptive mean shift (AMS) technique. The automatic CC contour initialization (ACI) is achieved using the region analysis, template matching and location analysis, thus identify the $C C$ region. Finally, the boundary of recognized $C C$ region is used as the initial contour in the Geometric Active Contour (GAC) model, and is evolved to obtain the final segmentation result of $C C$. Experimental results demonstrate that the proposed AMS-ACI technique is able to provide accurate initial CC contour, and the proposed AMS-ACI-GAC technique overcomes the problem of user-guided initialization in existing GAC techniques, and provides a reliable and accurate performance in CC segmentation.
\end{abstract}

Keywords:

Adaptive Mean Shift Clustering, Automated Segmentation, Corpus Callosum, Geometric Active Contour, Template Matching

\section{INTRODUCTION}

The corpus callosum (CC) is a large white-matter structure in human brain. It connects the left and right cerebral hemispheres, and transfers sensory, motor and cognitive information between the two hemispheres. Considering that human cognitive functions in brain are greatly impacted by the damaged interhemispheric communication, some neuropathological studies have been proposed to analyze the $\mathrm{CC}$ for subjects with neurological diseases [1-6]. Segmentation of CC is a fundamental step in the analysis of CC on brain medical images.

The in-vivo Magnetic Resonance Imaging (MRI) is a noninvasive medical imaging technique that provides high resolution images for the structure. According to the report of Canadian Institution of Health Information (CIHI), between 2011 and 2012, approximately 1.7 million MRIs were performed on Canadian patients, and the majority of MRI scans are for brain imaging [7]. With no radiation exposure to the subject, the MRI is regarded safer than other neuroimaging techniques, such as skull X-ray and computed tomography (CT) [9]. MRI images help to identify underlying structural abnormalities as causes of diseases.

Typically, in the T1-weighted midsagittal brain MRI slice, the $\mathrm{CC}$ has the appearance of a broad arched band. An example of the T1-weighted midsagittal brain MRI slice is shown in Fig.1(a), and an enlarged example of CC region is shown in Fig.1(b). Note that the yellow contours in both figures indicate the $\mathrm{CC}$ boundaries. It is observed that the $\mathrm{CC}$ appears as a bright region with narrow and long shape located near the centre of the brain. The location, size and shape of the CC region are important features for distinguishing the $\mathrm{CC}$ region from other brain tissue regions.



(a)



(b)
Fig.1. Corpus Callsoum in a T1-Weighted Midsagittal Brain MR Image (a) An Example of T1-Weighted Midsagittal Brain MRI Slice, the CC Region is highlighted by Yellow Contour (b) A Close up Example of the $\mathrm{CC}$, the Yellow Contour Indicates its Structure

Traditionally, the neuroimages are examined by professional radiologist, and the brain tissue and its change are manually separated from surrounding brain tissues based on their observation, knowledge and experience. Note that the accuracy for detection of epileptogenic regions using the neuroimaging techniques may be influenced by radiologist's subjective bias. Because of fatigue, overlook or overloaded images, epileptogenic regions with subtle structural changes might be missed. In addition, the inter-observer and intra-observer variations often occur [8]. In order to address these problems, it is of great value to develop an automated $\mathrm{CC}$ segmentation technique.

Segmentation of CC in midsagittal brain MRIs is a challenging and critical task in medical image analysis. The difficulties arise from many aspects, such as the low contrast of MRI scans and blurred CC boundaries. Several techniques have been proposed in the literatures for $\mathrm{CC}$ segmentation. Most of these techniques are based on Active Contour Model (ACM). Brejl et al. [9] proposed an automated model based technique for the segmentation of CC in sagittal brain MRIs. The technique first constructs shape model and boundary appearance model of $\mathrm{CC}$ from training images. Based on these two statistical models representing the $\mathrm{CC}$, a shapevariant Hough transform technique is applied to approximately localize the $\mathrm{CC}$, followed by an edge-based segmentation method. This technique does not need initial close-to-target localization. It is likely to fail, however, if a large variability exhibits in shape and edge appearance of the CC. Ginneken et al. [10] proposed an active shape model-based technique for the segmentation of $\mathrm{CC}$. This technique first calculates a set of optimal local features from the training images. A non-linear K-nearest neighbour classifier is 
then used to calculate the optimal displacements of landmarks. This technique has the limitation of model matching, and it cannot detect patterns which are not included in the given training images. Jacob et al. [11] proposed an Active Contour Model (ACM) based technique for CC segmentation. This technique uses a parameter independent gradient energy term, as well as an internal energy term which can ensure constant arc-length. The initialization of contour, however, is still required as user inputs, and the segmentation performance is easily influenced by the initialization of contour.

Some variants of Active Contour Model have also been proposed for CC segmentation in brain MR images. Sandhu et al. [12] proposed a Geometric Active Contour (GAC) technique for $\mathrm{CC}$ segmentation. The technique incorporates the probability density functions of the background and object pixels into active contour framework, and the evolution is steered by seeking the maximum distance of above two distributions. Although efficient CC segmentation results on brain MRIs have been achieved, initialization of the contour from user interaction is required at first. Zhou et al. [13] proposed an automated segmentation technique for rodent brain tissues in MRIs. This technique applies support vector machines (SVMs) to obtain prior shape knowledge of objects of interest, and incorporates an automatic shape selection into existing active shape model framework for Cerebellum, Neocortex, CC, External Capsule, Caudate Putamen, Hippocampus and Ventricles in MRIs. The technique is evaluated using a database of 5000 training images and 3250 test images, and an average overlap rate of $66.7 \%$ in segmentation of CC of test images was achieved. El-Zehiry et al. [14] proposed a technique to segment $\mathrm{CC}$ in the midsagittal MR images. The technique first extracts white matter using a hierarchical model combining active contour propagation and graph cut optimization. The connected component analysis is then used to segment the $\mathrm{CC}$ from the white matter. Lai et al. [15] proposed an automated technique to extract the boundary of CC on T1-weighted brain MRIs. The technique first calculates the Laplace-Beltrami (LB) Eigen function on the white matter surface, and then uses its zero level set curve to obtain the initial CC contour. Secondly, it deforms the initial curve to get the final curve with the geodesic curvature flow on the white matter surface.

Although the ACM based techniques are widely used for CC segmentation, and have been reported to provide a promising performance, the major limitation is the manual initialization of the CC boundary. The segmentation performance of ACM based technique is sensitive to the initial contour, and if the initial contour is far from the boundary of the object of interest, the ACM based technique is likely to fail. To address this limitation, we propose a hybrid technique for fully automated CC segmentation in this paper. The proposed technique uses an adaptive mean shift clustering technique which segments an image into several homogeneous regions, representing various brain tissues. The CC region is then detected based on region analysis and template matching, in conjunction with location analysis. Based on the obtained $\mathrm{CC}$ region, the boundary is extracted and evolved using a GAC model for the final segmentation of $\mathrm{CC}$ structure. The major contribution of the proposed technique is to provide an accurate initialization of the $\mathrm{CC}$ region, which results in a promising performance in terms of the segmentation accuracy and convergence speed.
The rest of the paper is organized as follows: Section 2 provides a review of GAC-based $\mathrm{CC}$ segmentation technique. Section 3 describes the details of the proposed technique. Section 4 evaluates the experimental results tested on real brain MRI databases, and the conclusions are presented in section 5 .

\section{BACKGROUND}

The GAC model has been shown to be very efficient in medical image segmentation, and has been used for $\mathrm{CC}$ segmentation. Therefore, we present a brief review of GAC-based $\mathrm{CC}$ segmentation in this section.

In the GAC framework, active contours are deformable curves that can be used to delineate the image structures. After the initialization of contour, the contour is allowed to evolve by seeking the minimization of an image-based energy functional [12], typically through gradient descent. It incorporates the region information into the GAC framework, and the energy functional uses the statistical information of image regions. In the GAC technique, the contour evolves by maximizing the distance between the intensity probability density functions of interior region and exterior region of contour. The active contour stops evolution when one of the following conditions is satisfied: (1) The evolution of contour comes to convergence; (2) The maximum number of iterations has been achieved. The schematic of the GAC-based CC segmentation technique is shown in Fig.2.

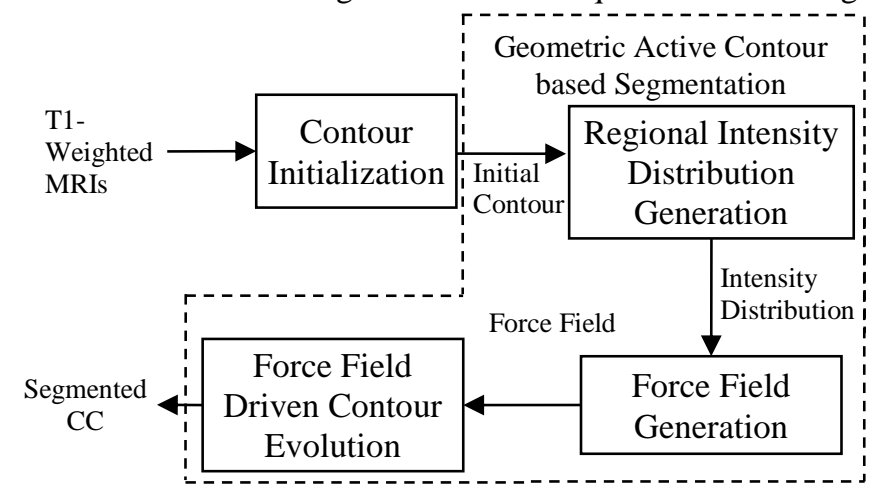

Fig.2. Simplified schematic of a GAC-based CC segmentation technique [12]

The four steps of the technique are as follows [12]:

a. Initial contour of the object is acquired from user interactions.

b. The probability density functions of intensities (histograms) are calculated for interior region of contour and exterior region of contour respectively.

c. The distance between two density functions are measured.

d. The contour evolves by seeking for the maximum of the distance between two distributions.

\section{THE PROPOSED TECHNIQUE}

The GAC technique reviewed in Section II, has a higher level of robustness to noise and initialization when compared with other ACMs. Similar to other ACMs, however, this technique also requires initialization of contour from users. In addition, it fails to 
detect the exterior and interior boundaries when the initial contour is far from the desired object boundary. To address these limitations, we propose a computer-aided technique for automated initialization of the contour based on the region, shape and location characteristics.

The schematic of the proposed technique is shown in Fig.3. It is observed that the proposed technique contains three modules: Adaptive Mean Shift Clustering, Automated CC Contour Initialization, and Geometric Active Contour based Segmentation. In the first module, a set of clusters are generated from an input image based on grayscale intensity closeness using the adaptive mean shift clustering. In the second module, area analysis, template matching, shape and location analysis are used to identify the specific cluster that contains $\mathrm{CC}$, and extract a rough boundary of $\mathrm{CC}$ as the initial contour. In the last module, a final $\mathrm{CC}$ boundary is calculated based on Active Contour Segmentation method. The details of these modules are presented in the following sections. A grayscale brain MR image as input is assumed in this paper.

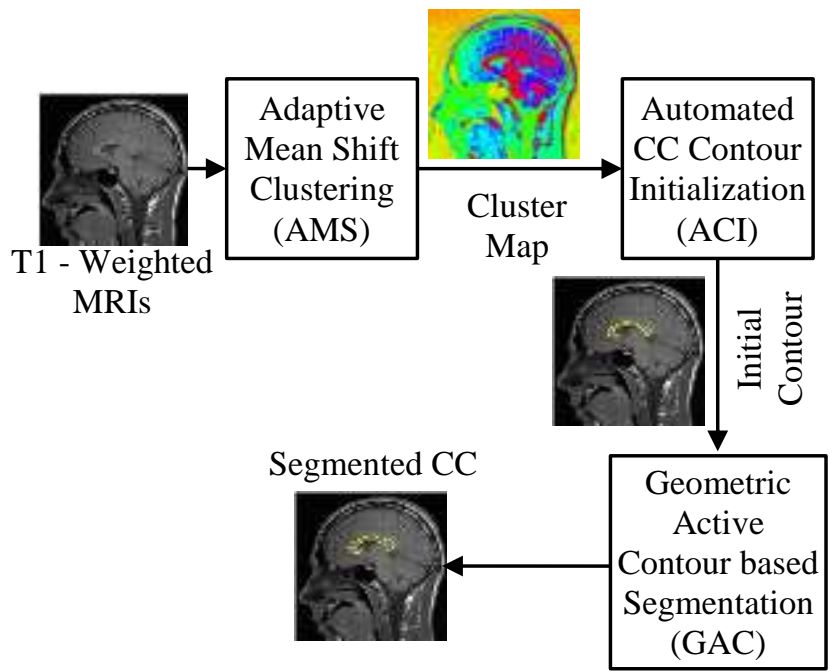

Fig.3. Schematic of the Proposed Technique

\subsection{ADAPTIVE MEAN SHIFT CLUSTERING (AMS)}

Based on observation in T1-weighted brain MRI (see Fig.1), the appearance of $\mathrm{CC}$ is generally homogenous (i.e., similar grayscale intensity). For accurate segmentation, it is helpful to apply an image clustering technique as the preprocessing step. In this work, the adaptive mean shift (AMS) technique [16] is applied with gray-level intensities features for image clustering.

The AMS technique [16] is an iterative technique that can be used for finding modes of the probability density function ( $p d f$ ) of the image intensity, and associating their neighboring grayscale intensities to the corresponding modes, thereby establishing clusters. Grayscale intensities associated with the same mode belong to the same cluster, and the number of clusters is the same as the number of modes. Prior knowledge of the clusters, e.g., the number of clusters, and the shape of clusters, are not required in the AMS technique [16]. In the $p d f$ of grayscale intensities of a brain MR image, there would be dense regions if this image has homogeneous areas. In other words, homogeneous areas in the image correspond to various segments in the $p d f$ of grayscale intensities. For example, if there is a large homogeneous area in a grayscale image, with an intensity of about 100 (in an 8-bit scale), it could lead to a segment in $p d f$ of grayscale intensities, around 100. The peak intensity of 100 is considered as a mode of the $p d f$ of grayscale intensities of this image.

Note that in AMS clustering, only the grayscale intensity information of the image is considered, and the spatial information is not considered. This is because the grayscale intensity is good enough for the AMS technique to generate a rough shape of CC region. In other words, the generated clusters would be similar in intensity, and it is possible that a cluster may include several unconnected regions in the image space.

There are seven major steps of AMS clustering, which are explained in the following.

Step 1: For a brain MR image $I$, the $p d f$ of the grayscale intensities is computed. Let $P_{i}$ denote the $i^{\text {th }}$ pixel in the image $I$, and $V_{i}$ denote the grayscale intensity of $P_{i}, 1 \leq i \leq N$, where $N$ is the total number of pixels in the image $I$. Note that $V_{i}$ is a mapping point of $P_{i}$ in the grayscale intensity feature space of the image.

Example of a T1-weighted brain MRI is shown in Fig.4(a), and its $p d f$ of grayscale intensities is shown in Fig.4(b), and its magnified $p d f$ of grayscale intensities in the range of $[50,120]$ are shown in Fig.4(c). It is observed in Fig.4(b) that several local peaks are present in the $p d f$ of the grayscale intensities. Some local peaks could be better observed in Fig.4(c). In other words, homogeneous areas with pixels that have similar grayscale intensities are present in the brain MRI. Our goal is to delineate clusters in the grayscale feature space, with the intention of clustering homogenous areas in the brain MRI. Denote the number of clusters in the image by $m$. Initially, $m$ is zero. To start with, a feature point $V_{c u r}$ is initialized as the smallest gray value in the image, and then go to step 2.

Step 2: For the point $V_{\text {cur }}$ (a gray intensity value), the $L 1$ distance (i.e., Manhattan distance) between $V_{\text {cur }}$ and its neighbors in the feature space is calculated, and the neighbors of $V_{c u r}$ are sorted by order of increasing distance magnitude to $V_{c u r}$. Let $V_{c u r, K}$ denote the $K^{\text {th }}$ - nearest neighbor of $V_{c u r}$. The adaptive bandwidth $h_{\text {cur }}$ for $V_{\text {cur }}$ (for a given $K$ value, $K=26,214$ ) is calculated as follows:

$$
h_{\text {cur }}=\left\|V_{c u r}-V_{c u r, K}\right\|_{1}=\left|V_{c u r}-V_{c u r, K}\right|
$$

where, $K$ is the number of neighbors that is considered in calculating the adaptive bandwidth $h_{c u r}$ for $V_{c u r}$. In this paper, we calculate $K$ using the following equation:

$$
K=p \cdot N
$$

where, $p$ is a pre-selected constant, $0 \leq \mathrm{p} \leq 1$.



(a) 


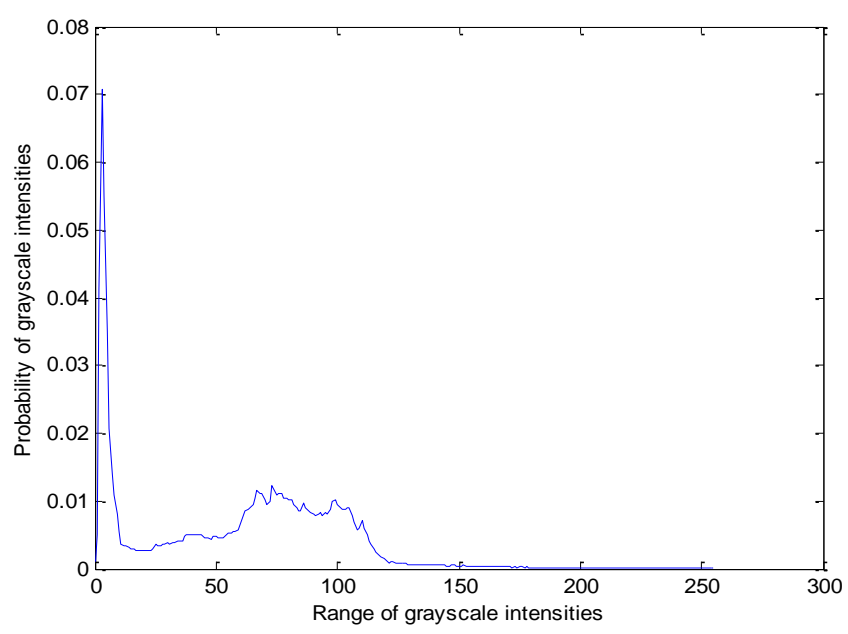

(b)

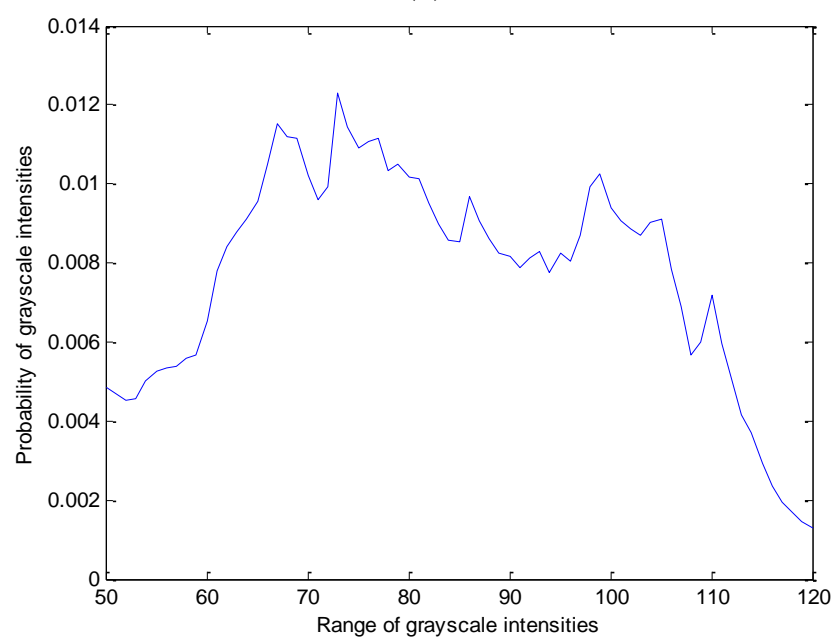

(c)

Fig.4. Example of a feature space. (a) A $512 \times 512$ brain MRI (b) $P d f$ of the gray intensity value of the image shown in (a) (c) A blown-up version of the $p d f$ shown in (b), in the range of [50 120]

Step 3: For the point $V_{\text {cur }}$, a symmetric window $S_{\text {cur }}$ with bandwidth $h_{\text {cur }}$ is generated, i.e., $V_{\text {cur }}$ is the centre of window $S_{\text {cur }}$. Let the number of points included in $S_{\text {cur }}$ be denoted by $J_{c u r}$ (including $V_{\text {cur }}$ ).

Step 4: The weighted mean shift vector $M_{h}\left(V_{c u r}\right)$ within the window $S_{c u r}$ is calculated using Eq.(3):

$$
M_{h}\left(V_{\text {cur }}\right)=\frac{\sum_{j=1}^{J_{c u r}} \frac{1}{h_{\text {cur }}^{d+2}}\left(V_{j}-V_{\text {cur }}\right) G\left(\frac{V_{j}-V_{\text {cur }}}{h_{\text {cur }}}\right)}{\sum_{j=1}^{J_{c u r}} \frac{1}{h_{\text {cur }}^{d+2}} G\left(\frac{V_{j}-V_{\text {cur }}}{h_{\text {cur }}}\right)}
$$

where, $d$ is the dimension of feature space (we have used $d=1$ in this work), and $V_{j}$ is the $j^{\text {th }}$ point within window $S_{\text {cur. }} M_{h}\left(V_{\text {cur }}\right)$ is the distance between the weighted mean of points (gray intensity values) within window $S_{\text {cur }}$ and the centre of window $V_{\text {cur }} . G(x)$ is the kernel function, and it is used for weights. In our work, the uniform kernel function is used, i.e., all point within window $S_{\text {cur }}$ are given the same weight. $G(x)$ is defined as follows:

$$
G(x)=\left\{\begin{array}{cc}
c & |x| \leq 1 \\
0 & \text { otherwise }
\end{array}\right.
$$

where, $c$ is a normalization constant such that $\int_{-\infty}^{+\infty} G(x) d x=1$.

Step 5: Determine if the following equation is satisfied,

$$
\left|M_{h}\left(V_{\text {cur }}\right)\right|<T_{A M S}
$$

where, $T_{A M S}$ is a predefined threshold used as a stopping criterion for search of a mode in the current neighborhood in feature space. If Eq.(4) is satisfied, the current $V_{c u r}$ is stored as the converged feature point, and then algorithm goes to step 6. Otherwise, the feature point $V_{\text {cur }}$ is updated as follows:

$$
V_{\text {cur }}=V_{\text {cur }}+M_{h}\left(V_{\text {cur }}\right)
$$

and the algorithm returns to step 2.

Step 6: The current converged point $V_{\text {cur }}$ is stored as a mode, and a new cluster by including $V_{\text {cur }}$ and all the points converging to $V_{\text {cur }}$ (i.e., all the points visited by AMS in steps 2-5) is generated. Let the generated cluster be denoted by $C_{m}$ (where $m$ is the cluster number).

Step 7: In this step, we first determine if there is any pixel in the image which falls outside all the clusters that have been generated so far. Let us denote the set of image pixels which are not among clusters $\left\{C_{1}, \ldots, C_{m}\right\}$ by $C_{r e m}$. If $C_{r e m}$ is a null set, the clustering stops. Otherwise, the feature point $V_{\text {cur }}$ is initialized as follows:

$$
V_{\text {cur }}=g
$$

where, $g$ is the smallest grayscale intensity in $C_{r e m}$, and the algorithm returns to step 2 .

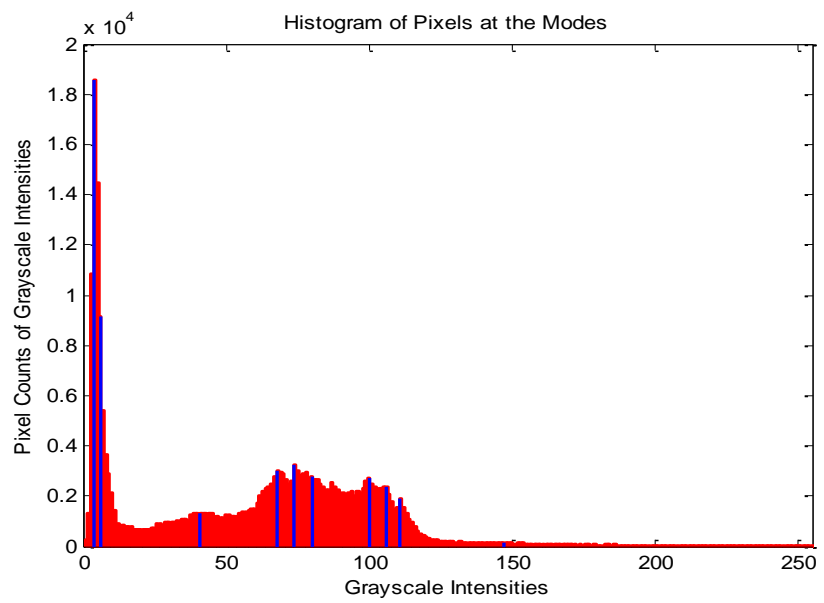

Fig.5. Histogram of modes in AMS clustering obtained for the image shown in Fig.4(a). There are 10 modes with values [4, 6 $41,68,74,80,100,106,111,147]$. The modes at 147 is not clearly visible, as there are only 117 pixels

An example of histogram of pixels at the modes is shown in Fig.5. In this example, there are 10 modes, and each mode corresponds to a cluster. The clusters can be seen more clearly (shown using different colors) in Fig.7(d).

The gray intensity of a mode corresponds to a representative intensity of a cluster pixel values. Examples of clusters generated in the $1^{\text {st }}$ AMS iteration and in the $10^{\text {th }}$ AMS iteration are shown as binary images in Fig.6(a) and Fig.6(b) respectively. In these 
binary images, white pixels indicate cluster points (pixels).

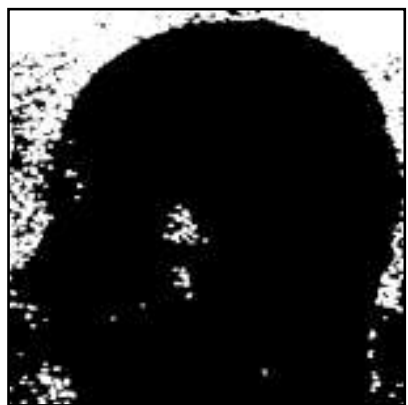

(a)

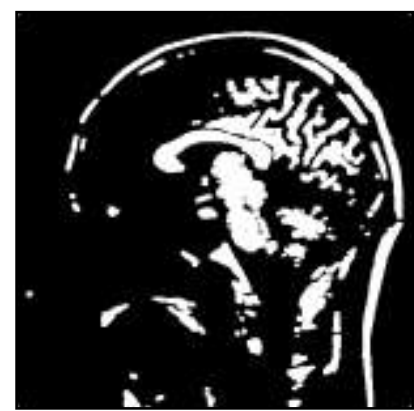

(b)

Fig.6. Examples of clusters (white pixels) generated in AMS iterations. (a) Cluster generated from Fig.4(a) in the $1^{\text {st }}$ AMS iteration. The final bandwidth $h_{i}=1$, and the cluster pixel value range is [15]. (b) Cluster generated from Fig.4(a) in the $10^{\text {th }}$ AMS iteration. The final bandwidth $h_{i}=108$, and the cluster pixel value range is $[122,256]$

As we defined previously (see Eq.(1)), $K$ is the number of neighbors that is considered in calculating the adaptive bandwidth for a feature point. The determination of $K$ depends on the choice of $p$ value (see Eq.(2)). The cluster performance is sensitive to the choice of $p$ value. An illustrative example of AMS clustering (with different $p$ value) is shown in Fig.7. In Fig.7, the cluster maps and histograms corresponding to the image in Fig.4 (a) (with $N=262,144$ ) are shown. The Fig.7(a), Fig.7(c) and Fig.7(e) show the cluster maps generated by the AMS, with $p=0.08, p=0.10$ and $p=0.12$, respectively. The cluster histograms corresponding to Fig.7(a), Fig.7(c) and Fig.7(e) are shown in Fig.7(b), Fig.7(d) and Fig.7(f), respectively. It is observed that with $p=0.08, p=$ 0.10 and $p=0.12$, AMS generates 12, 10 and 9 clusters, respectively.

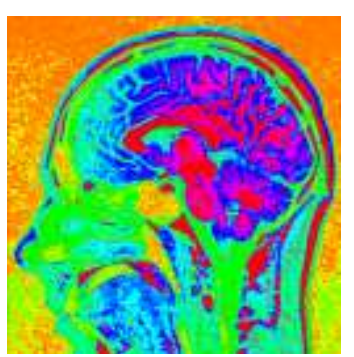

(a)

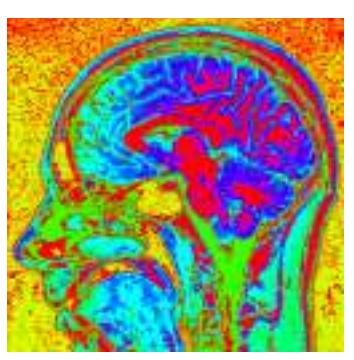

(c)

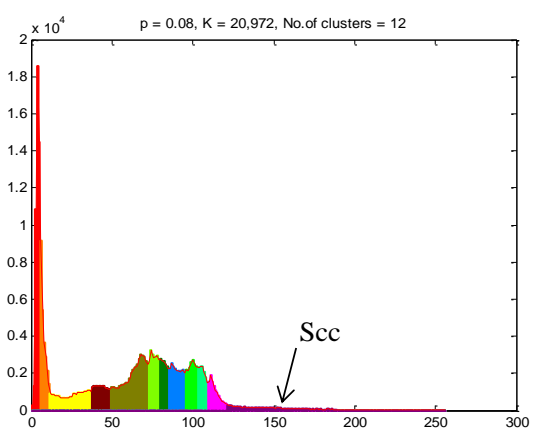

(b)

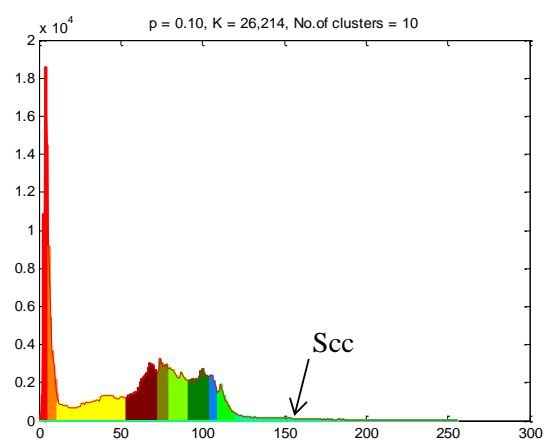

(d)

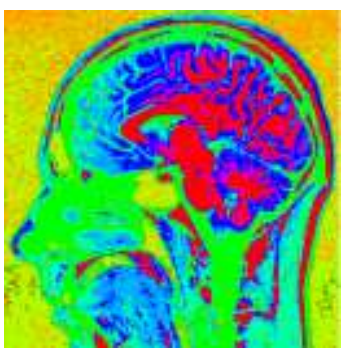

(e)

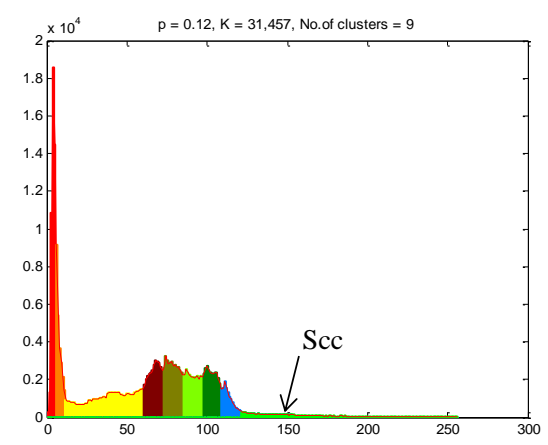

(f)
Fig.7.Cluster maps and histograms generated by AMS. (a)(c)(e) Cluster maps generated by AMS with $p=0.08, p=0.10$ and $p=$ 0.12 , respectively. (b)(d)(f) Cluster histograms corresponding to

$$
\text { (a) (c)(e) }
$$

The Fig.8(a), Fig.8(b) and Fig.8(c) show magnified CC clusters (in red color) corresponding to Fig.7(a), Fig.7(c) and Fig.7(e), respectively. Let $S_{C C}$ denote the cluster that includes the $\mathrm{CC}$ region. It is observed that, with $p=0.08$ (see Fig.8(a)), $S_{C C}$ roughly has the shape of the $\mathrm{CC}$ region, although there are some unconnected parts inside the $\mathrm{CC}$ region. With $p=0.10$ (see Fig.8(b)), the $S_{C C}$ follows the shape of the CC region more closely, and also the unconnected parts inside the $\mathrm{CC}$ region have been eliminated. On the other hand, with $p=0.12$ (see Fig.8 (c)), the $S_{C C}$ cannot keep the rough shape of the CC region. In other words, $p=0.10$ provides the best clustering performance.

Another example of cluster maps and histograms generated by AMS with $p=0.08, p=0.10$ and $p=0.12$ are shown in Fig.9. For this image also, $p=0.10$ provides the best performance. Based on our experimental results, we therefore select $p=0.10$ to obtain a good initial cluster map generation for CC segmentation.

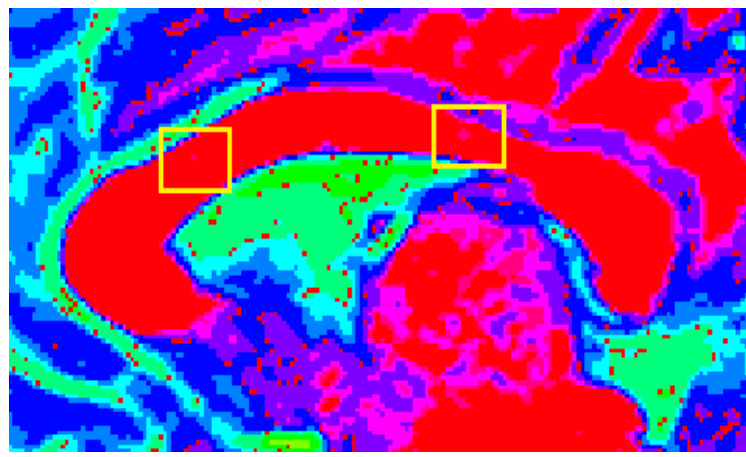

(a)

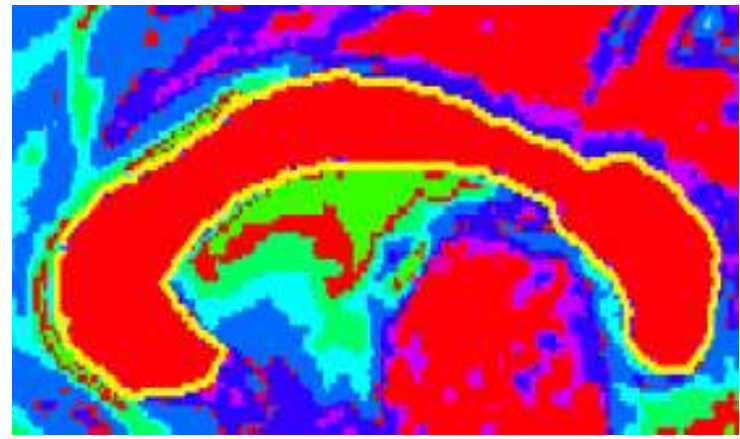

(b) 


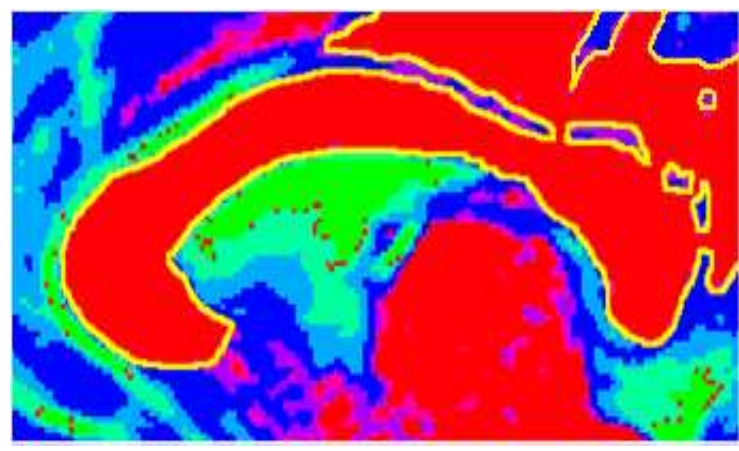

(c)

Fig.8. Magnified CC Clusters. (a) is for Fig.7(a), and the yellow rectangles indicate the unconnected parts inside the CC. (b) is for Fig.7(c), and (c) is for Fig.7(e), and the yellow contour indicate the boundary of connected parts inside the $\mathrm{CC}$ and around the $\mathrm{CC}$

\subsection{AUTOMATED CC INITIALIZATION (ACI)}

CONTOUR

Given the cluster map generated by the AMS technique, a series of identification steps are now used to identify the cluster that contains the CC structure. The details of this model are presented below.

\subsubsection{Region Analysis:}

Considering that the $\mathrm{CC}$ is the largest white matter tissue in the brain, we perform a region analysis technique, which is capable of detecting the candidate $\mathrm{CC}$ clusters from all generated clusters by the AMS technique. Based on the prior knowledge, the number of pixels that are included in the $\mathrm{CC}$ cluster is assumed to be larger than a predefined threshold $T_{T h r}$. Based on experimental results, we set $T_{T h r}=0.01 N$, where $N$ is the total number of pixels in the brain MRI. The fractions 0.01 are determined based on the domain prior and experimental results. To be specific, let the $i^{\text {th }}$ cluster obtained from AMS Clustering be denoted by $C_{i}$, the candidate $\mathrm{CC}$ clusters be denoted by $C l_{\text {cand }}$, and the number of pixels included in a cluster be denoted by $P_{C}$. The $C_{i}$ is determined as follows:

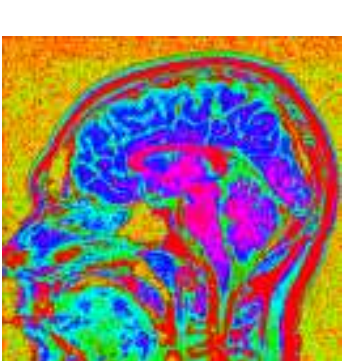

(a)

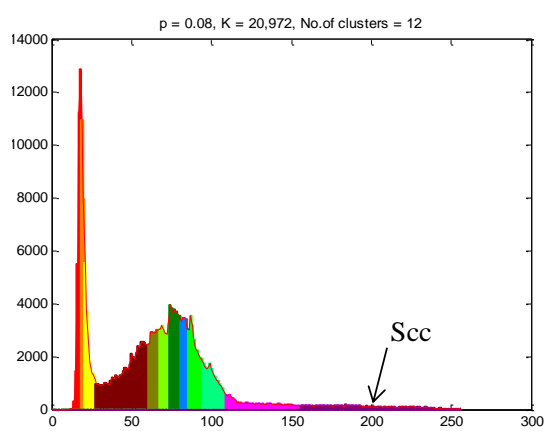

(b)

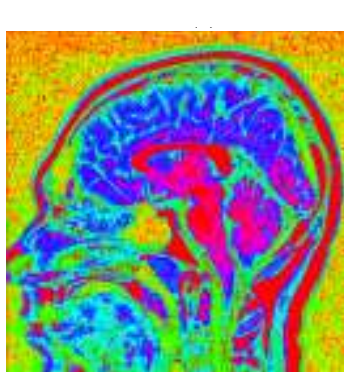

(c)

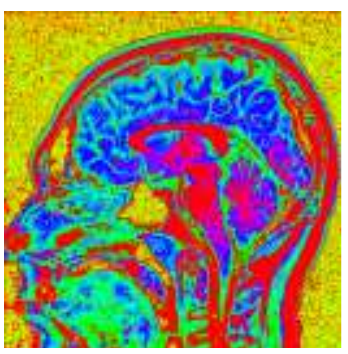

(e)

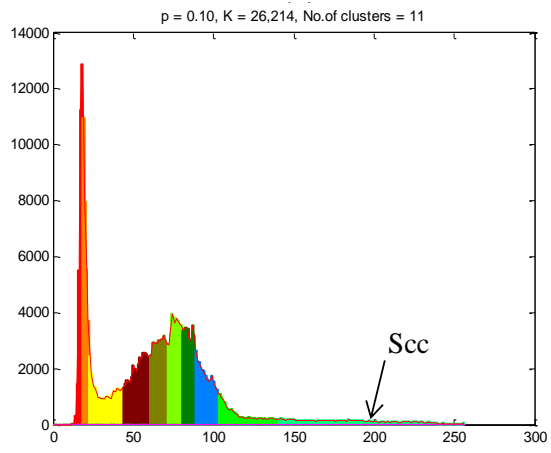

(d)

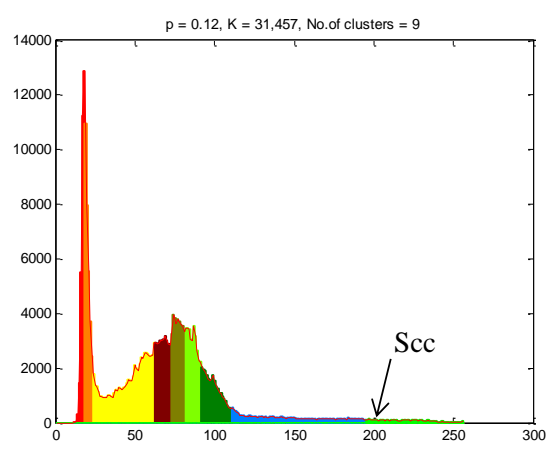

(f)
Fig.9. Cluster maps and histograms generated by AMS. (a)(c)(e) Cluster maps generated by AMS with $p=0.08, p=0.10$ and $p=$ 0.12 , respectively. (b)(d)(f) Cluster histograms corresponding to (a) (c) (e)

$$
C_{i}=\left\{\begin{array}{cll}
C l_{\text {Cand }}, & \text { if } & P_{C} \geq T_{T h r} \\
\text { others } & & \text { otherwise }
\end{array}\right.
$$

By applying the region criterion to the pre-clustered regions, we obtain some binary images with a few candidate regions. In this paper, let this binary image be denoted by $I_{B}$. An example of region analysis results corresponding to Fig.7(c) is shown in Fig.10, where there are two candidates satisfying the area criteria (the white regions indicate the candidate cluster regions). Based on the observation of CC on brain MRI, the appearance of CC is much brighter than the appearance of background, in other words, the intensity of $\mathrm{CC}$ is much higher than the intensity of background. Therefore, only the cluster with higher intensity would be selected as a CC cluster.

\subsubsection{Template Matching:}

As shown in Fig.10, besides cluster regions containing the object of interest (true positive), a few other cluster regions (false positive) exist. Therefore, in this step, the template matching (TM) technique is applied to detect the $\mathrm{CC}$ region and eliminate the false positives. This step can be divided into several sub steps which are presented as follows:

Step 1: In the first step, a set of template images is generated based on a $\mathrm{CC}$ structure obtained from a healthy person. Based on the knowledge of CC, a template image database is generated with templates of various sizes, shear and orientations applying appropriate geometric transforms. The scaling parameters $\left[S_{x}, S_{y}\right]$, clockwise rotation angle $\theta$ and shear transform parameters [Sh, $S h_{y}$ ] are incorporated using the following coordinate substitutions: 


$$
\left[\begin{array}{l}
x^{\prime} \\
y^{\prime}
\end{array}\right]=\left[\begin{array}{cc}
s_{x} & 0 \\
0 & s_{y}
\end{array}\right]\left[\begin{array}{cc}
\cos \theta & \sin \theta \\
-\sin \theta & \cos \theta
\end{array}\right]\left[\begin{array}{cc}
1 & s h_{y} \\
s h_{x} & 1
\end{array}\right]\left[\begin{array}{l}
x \\
y
\end{array}\right]
$$

where, $x$ and $y$ respectively denote the horizontal and vertical coordinates of a pixel. In this work, the parameter settings are as follows: (i) $S_{x}, S_{y} \in\{0.8,0.9,1\}$; (ii) clockwise rotation angle $\theta$ $\in\left\{-30^{\circ},-15^{\circ}, 0^{\circ}, 15^{\circ}\right\}$; (iii) $S h_{x}, S h_{y} \in\{0,0.05,0.1,0.15\} .48$ templates are included in the template image dataset. Examples of $\mathrm{CC}$ template images are shown in Fig.11.

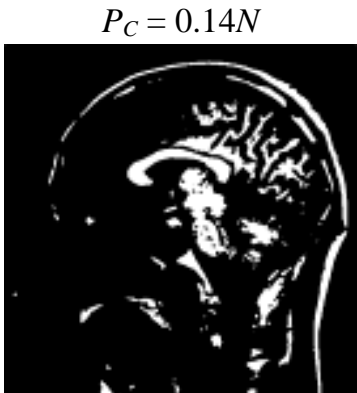

(a)



(b)

Fig.10. Examples of area analysis results. (a) The largest cluster $P_{10}$ with $P_{C}=0.14 N$ (having grayscale values in the range of [122,256]), (b) The second largest cluster $P_{2}$ with $P_{C}=0.14 \mathrm{~N}$ (having grayscale values in the range of $[6,11]$ )
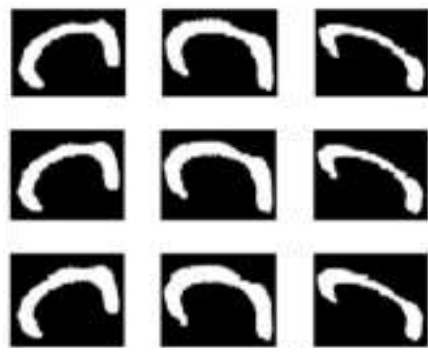

(a)
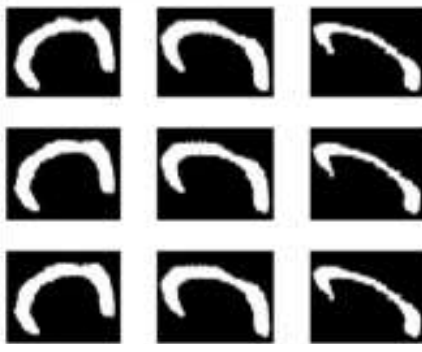

(b)
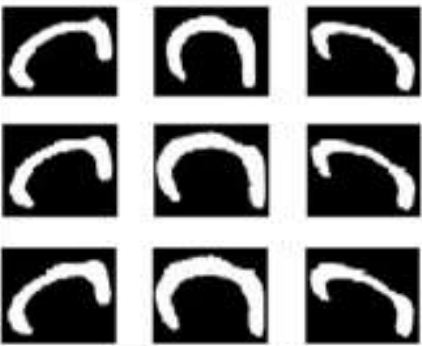

(c)

Fig.11. Examples of CC templates with parameters $S_{x}, S_{y} \in$

$\{0.8,0.9,1\}, \theta \in\left\{-30^{\circ},-15^{\circ}, 0^{\circ}, 15^{\circ}\right\}$, (a) $S h_{x}, S h_{y} \in\{0.05\}$

(b) $S h_{x}, S h_{y} \in\{0.1\}$ (c) $S h_{x}, S h_{y} \in\{0.15\}$

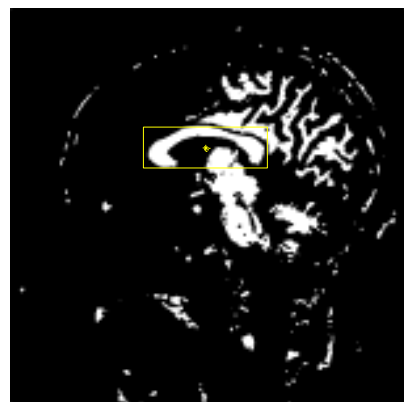

(a)

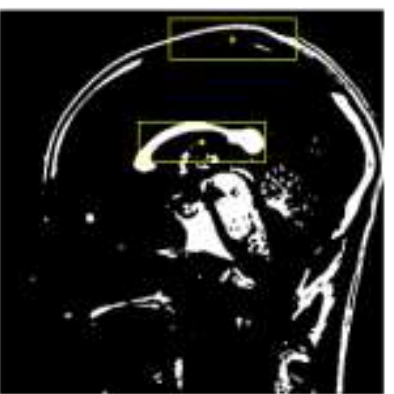

(c)

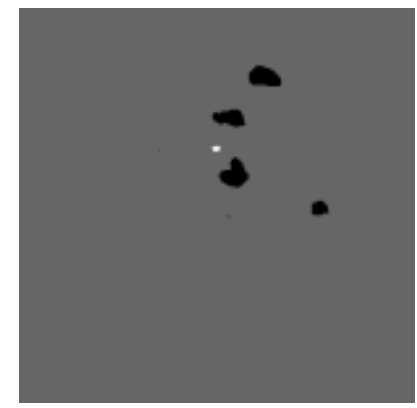

(b)

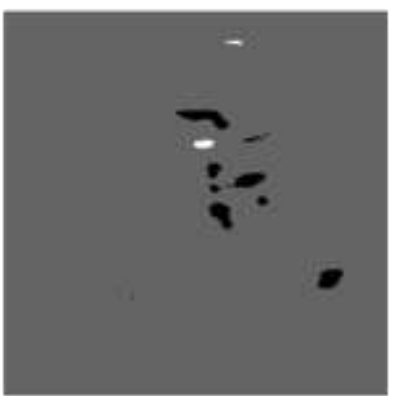

(d)
Fig.12(a)(c) Two examples of template matching results, the yellow rectangles indicate the detected regions. (b)(d) The corresponding NCC images of $(\mathrm{a})(\mathrm{c})$

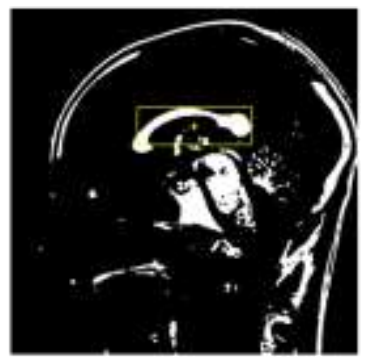

(a)

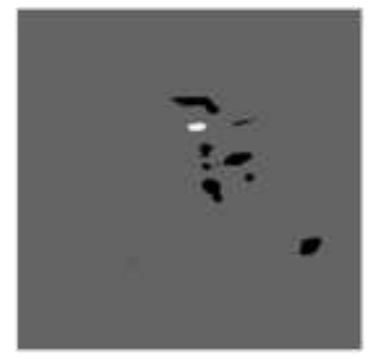

(b)
Fig.13. An example of shape and location analysis result, the yellow rectangles indicate detected regions. (b) The corresponding NCC images of (a)

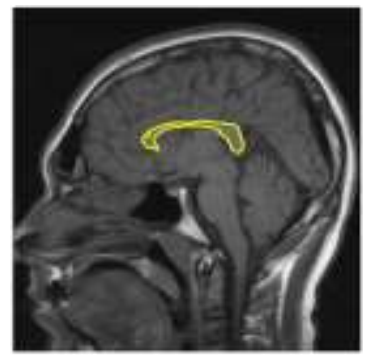

(a)

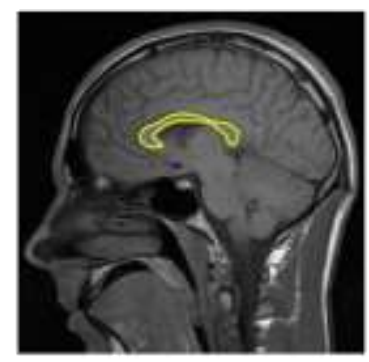

(b)
Fig.14. Examples of initial contour of CC, the yellow contour indicates the generated initial contour of $\mathrm{CC}$

Step 2: Given the dataset of $\mathrm{CC}$ templates with various sizes, orientations and shapes, each CC template is translated to every possible location in the binary image $I_{B}$. The similarity between a template $t$ and a sub-image $f$ is measured by calculating the value 
of normalized cross correlation (NCC) [17], which is defined as:

$$
\gamma(u, v)=\frac{\sum_{x, y}\left(f(x, y)-\bar{f}_{u, v}\right)(t(x-u, y-v)-\bar{t})}{\sqrt{\sum_{x, y}\left(f(x, y)-\bar{f}_{u, v}\right)^{2} \sum_{x, y}(t(x-u, y-v)-\bar{t})^{2}}}
$$

where, $f(x, y)$ is the pixel in the sub-image, $(u, v)$ is the center of sub-image, $\bar{f}_{u, v}$ is the mean of sub-image, whereas $t(x-u, y-v)$ is a pixel in the translated template, and $\bar{t}$ is the mean of template. The value of NCC is between 0 and 1 , and a higher value indicates higher similarity. By translating each template on the image $I_{B}$, we obtain the response in mage with NCC values.

Step 3: A block centered at $(u, v)$ is a candidate of $\mathrm{CC}$, when satisfying the following condition:

$$
\gamma(u, v)>T_{N C C}
$$

where, $T_{N C C}$ is a predefined threshold. In this work, $T_{N C C}$ is empirically set to 0.7. Examples of two template matching results is shown in Fig.12(a)(c), and their corresponding NCC images are shown in Fig.12(b)(d). The brightest part reflects regions with $\gamma \geq$ 0.7 , the gray part reflects regions with $0.4<\gamma<0.7$, and the black part reflects regions with $\gamma \leq 0.4$. It is noticeable that the spurious candidate cluster as shown in Fig.12(c) has been filtered out.

\subsubsection{Location Analysis:}

As shown in Fig.12 (c), besides cluster regions containing the object of interest, spurious CC clusters may be observed. Therefore, in this step, the supplementary analysis is required to further improve the initialization performance. In the proposed technique, location analysis of candidate regions are performed, and several sub steps are presented as follows:

Step 1: Note that the CC structure is usually located closer to the center of the brain MR images, compare with the top skull. In order to ignore the effect of image size, the location feature can be defined by the distance $\left(l_{d}\right)$ between candidate cluster region center and the image center to the image height $\left(l_{h}\right)$ ratio.

Step 2: For a candidate cluster region, we use the location criteria based on the prior domain knowledge to determine whether it is a CC structure or spurious CC structure.

$$
A=\left\{\begin{array}{cc}
C C \quad \text { if } l_{d} / l_{h} \leq T_{L} \\
\text { spurious CC } \text { otherwise }
\end{array}\right.
$$

Threshold $T_{L}$ is set to 0.25 empirically to make sure that the CC structure is located near the image center. Furthermore, extract the largest connected region $A$ (with largest number of pixels) in the detected region. An example of shape and location analysis result has been shown in Fig.13. It is noticeable that the spurious candidate cluster as shown in Fig.12(c) has been filtered out.

Step 3: The boundary of the CC region detected in step 2 is extracted as the initial contour of CC. Two examples are illustrated in Fig.14.

\subsection{GEOMETRIC ACTIVE CONTOUR BASED SEGMENTATION (GAC)}

In Section 3.2, the initial contour of CC region is extracted using a hybrid initialization technique, and this close-to-target initialization will facilitate the quick evolution of contour in the GAC-based technique [12] for CC segmentation. In this section, the final segmentation result of $\mathrm{CC}$ region is captured by the GAC technique [12].

Let $C$ denotes the initial contour of $\mathrm{CC}$ region, and $C$ represents a set of zeros of a signed distance function $\phi: \mathfrak{R}^{2} \rightarrow \mathfrak{R}$, let the input image be defined on the domain $\Omega$, i.e.

$$
\left\{\begin{array}{c}
C=\{x \in \Omega: \phi(x)=0\}, \\
\text { interior }(C)=\{x \in \Omega: \phi(x)<0\}, \\
\text { exterior }(C)=\{x \in \Omega: \phi(x)>0\} .
\end{array}\right.
$$

The initial contour $C$ evolves in the hope that the interior region of $C$ matches the $\mathrm{CC}$ region, and the exterior region of $C$ matches the background. In the GAC-based technique [12], the image intensity probability density functions of the background and object are incorporated into the process of contour evolution.

Considering that the gray scale intensities of the image are the random variable $\mathrm{z} \in \mathrm{Z}$. Therefore, the probability density functions (PDFs) $p_{\text {in }}(z, \phi)$ and $p_{\text {out }}(z, \phi)$ of intensities in the interior and exterior of the contour $C$ can be evaluated respectively.

In the GAC model [12], the similarity between intensity distributions interior and exterior of the contour $C$ is measured by the standard deviation between the log-likelihood of $p_{i n}(z, \phi)$ and $p_{\text {out }}(z, \phi)$. It is assumed that there is a distinct difference between the intensity distribution in the interior and exterior of the contour. Based on this assumption, the evolution of the contour would be driven in finding the maximum similarity between $p_{i n}(z, \phi)$ and $p_{\text {out }}(z, \phi)$. To maximize the above similarity, the contour $C$ is evolved iteratively using the image energy function $E(z, \phi)$ defined as follows:

$$
E(z, \phi)=\sqrt{\varepsilon\left[\left(\log \frac{p_{\text {in }}(z, \phi)}{p_{\text {out }}(z, \phi)}\right)^{2}\right]-\varepsilon\left[\left(\log \frac{p_{\text {in }}(z, \phi)}{p_{\text {out }}(z, \phi)}\right)\right]^{2}}
$$

where, $\varepsilon[f(z)]$ denotes the expected value of the functional $f(z)$. The value of $z$ is restricted to a set of grayscale intensity values $\{1,2, \ldots, 256\} . p_{\text {in }}(z, \phi)$ and $p_{\text {out }}(z, \phi)$ are the $p d f$ s defined on the variable $z$. The evolution of contour $C$ (or equivalent $\phi$ ) is performed according to the equation,

$$
\frac{\partial \phi}{\partial t}=\nabla_{\phi} E(z, \phi)
$$

The evolution is stopped if the maximum number of iterations has been achieved.

\section{PERFORMANCE EVALUATION}

In this section, we first present the MRI databases and evaluation metrics used in the experiments. The selection of parameters is discussed. The performance of the proposed technique is then evaluated and compared with existing techniques.

\subsection{MRI DATA AND EVALUATION METRICS}

The brain MRI data were chosen from two brain MRI databases: 1) brain MRI (BMRI) database from the University of Alberta Hospital; 2) a publicly available Open Access Series of Imaging Studies (OASIS) MRI database [18]. The BMRI database contains 7 images from patients with neurological 
disorders, and the OASIS database contains 27 normal images from subjects in the age range 20 to 40 .

The ground truths of $\mathrm{CC}$ regions of images in the BMRI database are manually drawn by a professional neurologist from the University of Alberta Hospital. The ground truths of CC regions of images in the OASIS database are manually drawn according to medical knowledge learnt from the professional neurologist. The segmentation results are compared with the ground truths.

The segmentation results are compared with the ground truth data. To evaluate the segmentation performance, Accuracy, Sensitivity, F1-score and Time-cost are considered as evaluation metrics. The calculation of Accuracy, Sensitivity and F1-score are defined as follows [19]:

$$
\begin{aligned}
& \text { Accuracy }=\frac{T P}{T P+F P} \times 100 \% \\
& \text { Sensitivity }=\frac{T P}{T P+F N} \times 100 \% \\
& F 1=\frac{2 T P}{2 T P+F P+F N} \times 100 \%
\end{aligned}
$$

where, $T P$ is the number of pixels in the true positive area (image region correctly classified as $\mathrm{CC}$ ), $T N$ is the number of pixels in the true negative area (image region which is correctly classified as background), $F P$ is the number of pixels in the false positive area (image region which is incorrectly classified as CC), and $F N$ is the number of pixels in the false negative area (image region which is incorrectly classified as background). The illustration of $T P$ (in blue), TN (in gray), FP (in yellow), $F N$ (in green) is shown in Fig. 15.

An important parameter in the proposed segmentation technique is the NCC threshold $T_{N C C}$. The Fig.16(a) shows the maximum NCC values in the CC region of all 34 images, and it is observed that the NCC values fluctuate between 0.7 and 0.9 . The Fig.16(b) shows the segmentation performance (accuracy, sensitivity and F1-score) for various $T_{N C C}$ values, and $T_{N C C}=0.7$ is shown to provide the best performance. Therefore, in this paper, we have chosen $T_{N C C}=0.7$. Another parameter is the location threshold $T_{l}$. As the ratio of $l_{d}$ and $l_{h}$ for each image is very stable at about $0.2 . T_{l}$ is set to 0.25 to include all $\mathrm{CC}$ regions and, at the same time, exclude the upper skull regions.



Fig.15. The distribution of $T P$ (in blue), $T N$ (in gray), $F P$ (in yellow), $F N$ (in green). The black contour indicates the ground truth, and the red contour indicates the segmentation result

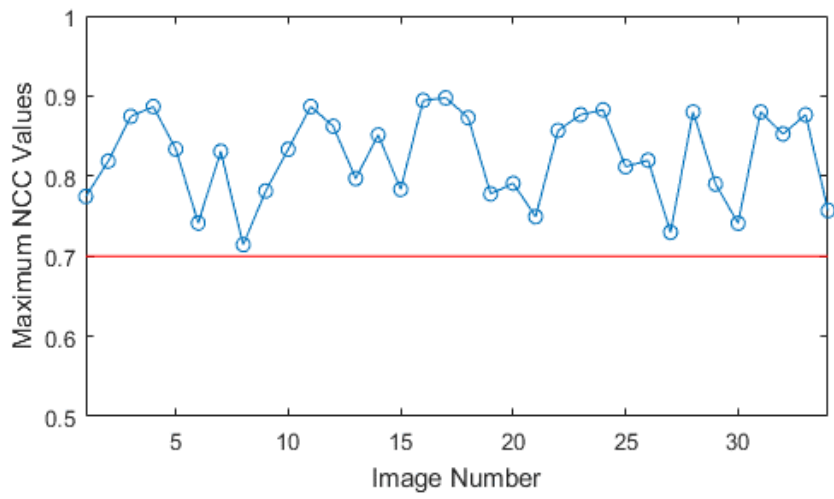

(a)

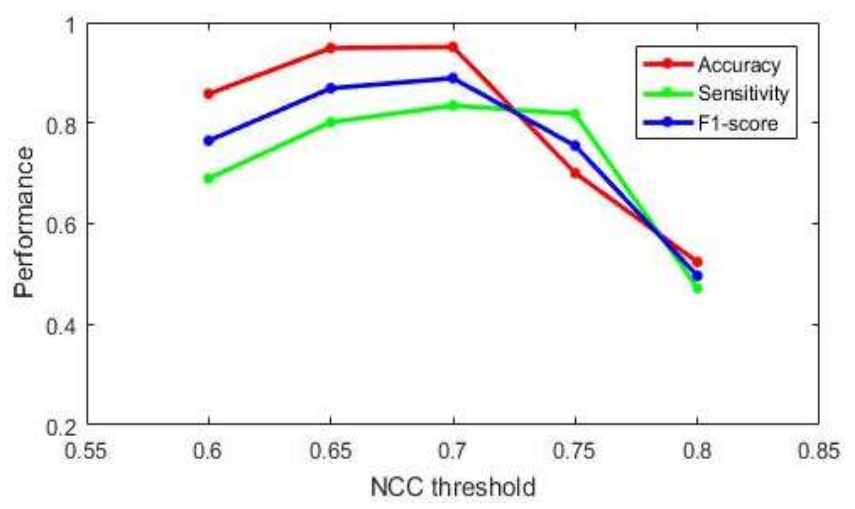

(b)

Fig.16. Selection of NCC threshold. (a) NCC values over images in the testing data set. (b) Segmentation performance against various NCC threshold values

\subsection{EXPERIMENTAL RESULTS}

We now evaluate the segmentation performance of the proposed AMS-ACI-GAC segmentation technique, and compare it with the AMS-ACI, and GAC technique [12]. The initial contour of the GAC technique [12] is a quadrilateral that is manually placed on the location of $\mathrm{CC}$. In the experiment, the principle of initial contour design is that the contour would include the $\mathrm{CC}$ region as much as possible and include surrounding regions as less as possible. Two examples of initial contour of typical GAC technique [12] are shown in Fig.17.

The classification performance of the $\mathrm{CC}$ pixels of the proposed AMS-ACI-GAC technique of a randomly selected image is shown in Table 1. The confusion matrix shows that 608 CC pixels (out of 3472 pixels) are classified as BG pixels, resulting in a sensitivity of $82.5 \%$.

The average evaluation results Accuracy, Sensitivity, F1-score for the MRI databases used in our experiment are shown in Table.2. The results in the table are shown as (mean, standard deviation). It is observed that the overall performance of the proposed technique is superior compared to the other two segmentation techniques. For example, the accuracy of the proposed AMS-ACI-GAC technique (95\%) may be marginally smaller compared to the AMS-ACI technique (98\%), but the sensitivity of the proposed technique is much higher. In other words, the AMS-ACI is able to provide a close-to-target initial contour, and the GAC technique is able to provide a more accurate final contour. The proposed AMS-ACI-GAC technique 
outperforms both GAC and AMS-ACI techniques in F1-score. For example, while the average $\mathrm{F} 1$-score of $\mathrm{CC}$ segmentation is $85 \%$ using the typical GAC technique, the F1-score of the AMSACI-GAC technique is $88 \%$, which indicate that the proposed technique has higher true positive rate.

Table.1. Confusion matrix using the proposed segmentation techniques. CC: corpus callosum. BG: background

\begin{tabular}{|c|c|c|c|}
\hline \multicolumn{2}{|c|}{} & \multicolumn{2}{c|}{ Ground truth } \\
\cline { 2 - 4 } \multicolumn{2}{|c|}{} & CC & \multicolumn{1}{c|}{ BG } \\
\hline $\begin{array}{c}\text { Segmentation } \\
\text { Result }\end{array}$ & CC & $\mathrm{TP}=2864$ & $\mathrm{FP}=207$ \\
\cline { 2 - 4 } & BG & $\mathrm{FN}=608$ & $\mathrm{TN}=258465$ \\
\hline
\end{tabular}

The proposed technique has been implemented in Matlab version R2011a on a computer with Intel Core i7-3667U CPU $2 \mathrm{GHz}$ and $8 \mathrm{~GB}$ RAM. The last column in Table. 1 shows the average execution time for an image. It is observed that the GAC technique [12] is slower by 40 times compared to the proposed AMS-ACI technique, and is about 4 times slower compared to the proposed AMS-ACI-GAC technique. This is primarily because the initial contour obtained by AMS-ACI technique is closer to
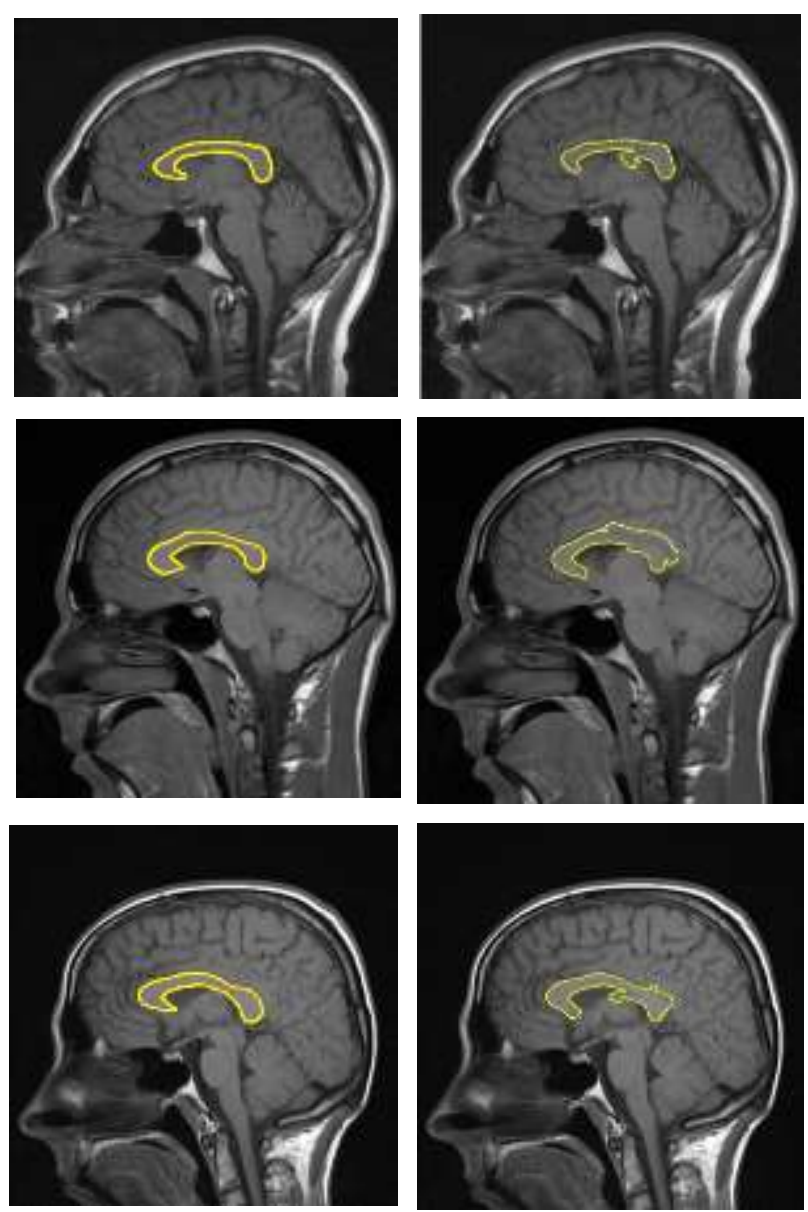

the actual $\mathrm{CC}$ contour, and hence the following GAC technique requires less time to converge. Therefore, the proposed segmentation technique is more suitable for real-time operation.
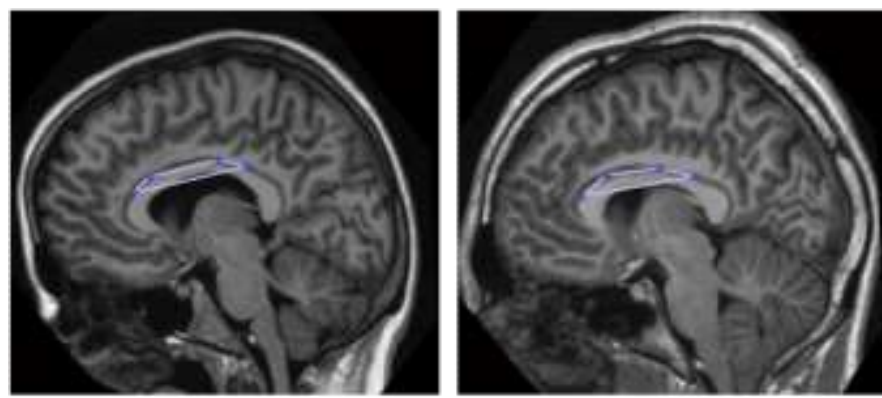

Fig.17. Initial Contours of typical GAC technique [12] in the experiments

The Fig. 18 presents examples of comparison between ground truth and segmentation results based on AMS-ACI technique, AMS-ACI-GAC technique and typical GAC technique [12]. It is observed that the AMS-ACI provides close-to-target initial contour, and the AMS-ACI-GAC technique gives more accurate results with respect to the ground truth.
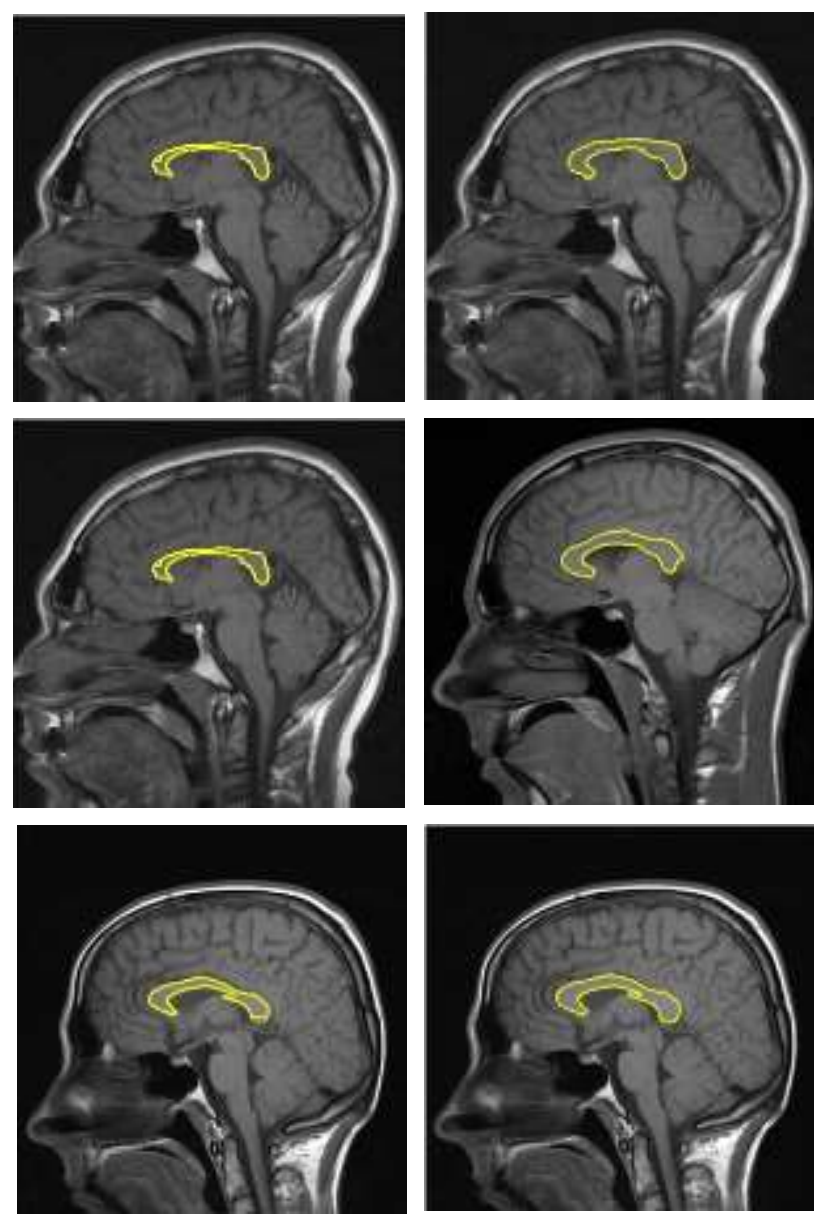

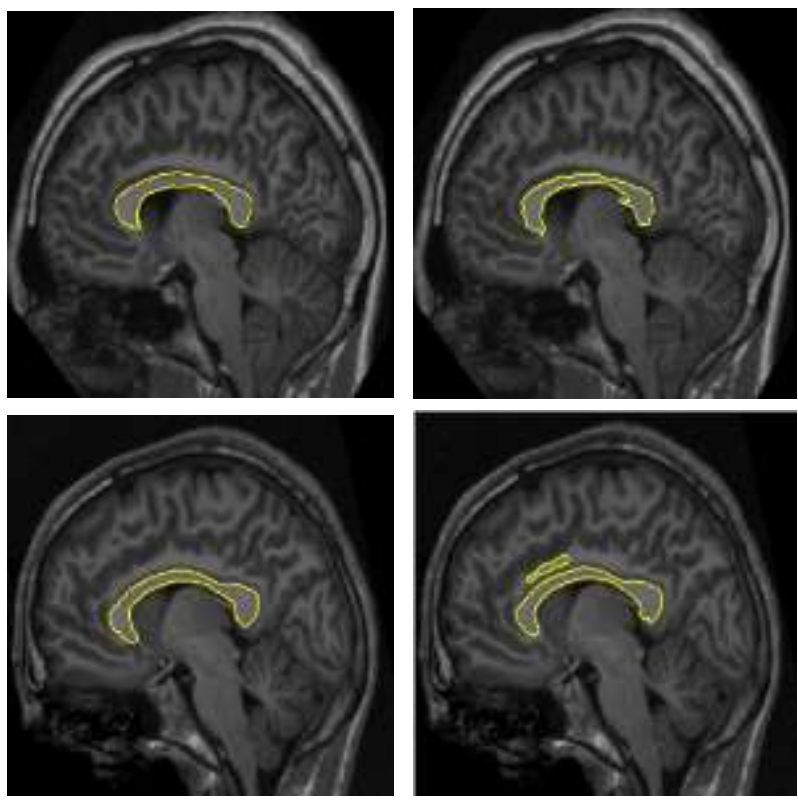

(a)

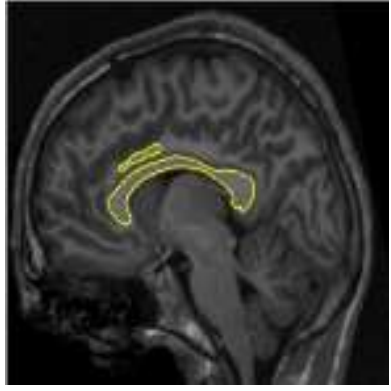

(b)
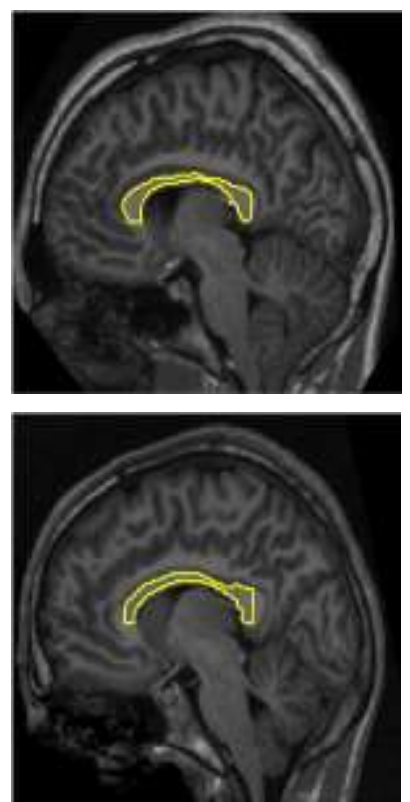

(c)
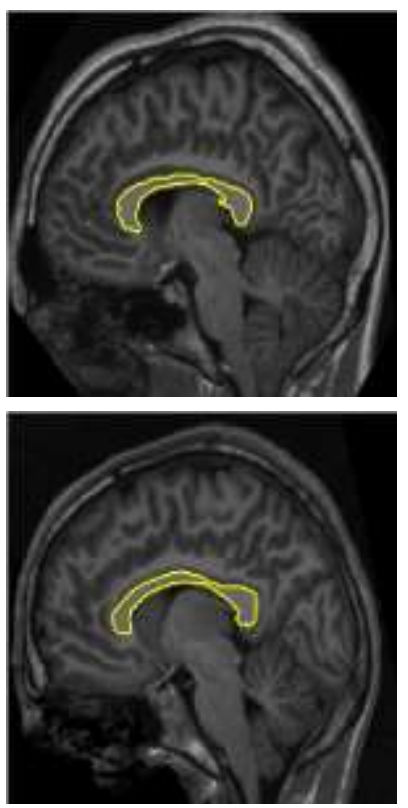

(d)

Fig.18. Comparisons of segmentation results (yellow contour) between ground truth, typical GAC [12], proposed AMS-ACI and AMSACI-GAC on T1-weighted midsagittal brain MR images. (a) The ground truths. (b) Segmentation results using typical GAC [12]; (c)

Segmentation results using proposed AMS-ACI technique; (d) Segmentation results using proposed AMS-ACI-GAC technique

Table.2. Comparison of the proposed segmentation technique with existing techniques.

\begin{tabular}{|c|c|c|c|c|}
\hline $\begin{array}{c}\text { Segmentation } \\
\text { Techniques }\end{array}$ & Accuracy & Sensitivity & F1 score & $\begin{array}{c}\text { Run-Time } \\
\text { (in sec) }\end{array}$ \\
\hline GAC [12] & $(0.86,0.16)$ & $(0.83,0.06)$ & $(0.85,0.08)$ & $(82.43,11.27)$ \\
\hline AMS-ACI & $(0.98,0.04)$ & $(0.66,0.09)$ & $(0.79,0.06)$ & $(2.27,0.53)$ \\
\hline $\begin{array}{c}\text { AMS-ACI- } \\
\text { GAC } \\
\text { (proposed) }\end{array}$ & $(0.95,0.09)$ & $(0.84,0.08)$ & $(0.88,0.04)$ & $(24.72,1.69)$ \\
\hline
\end{tabular}

The segmentation is based on homogeneity of regions, and the AMS may generate a cluster of $\mathrm{CC}$ with surrounding tissues. The Fig. 19 shows an example where the similar intensity values of neighboring tissue lead to incorrect segmentation (the missegmented region surrounded by the red rectangle in Fig.19(b)). In future, techniques to separate connected regions or shape priors may be considered to solve this issue and further improve the proposed technique.

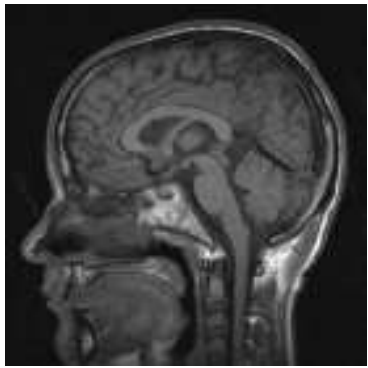

(a)

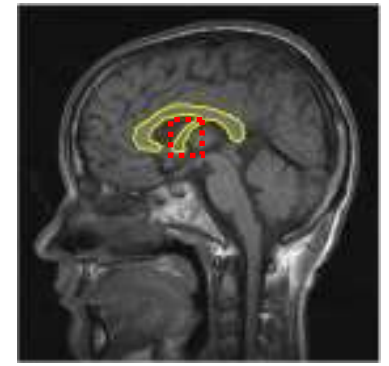

(b)
Fig.19. An example of false segmentation. (a) Input image. (b) Segmentation result (yellow contour) with false segmentation in red rectangle

\section{CONCLUSION}

This paper presents an efficient and novel technique for the automated segmentation of CC on T1-weighted midsagittal brain MRIs. First, the initial brain MRI is clustered into various homogeneous regions using the adaptive mean shift technique, represent various brain tissues. Second, the region analysis, template matching, combined with the shape and location analysis, are adopted to localized the $\mathrm{CC}$ region from all clusters generated by the adaptive mean shift technique, and the boundary of obtained CC region is extracted as the initial contour of the subsequent deformation model. Finally, the segmentation of CC is generated using the Geometric Active Contour (GAC) model [12]. The proposed technique has achieved the accuracy of $94.9 \%$, sensitivity of $83.6 \%, \mathrm{~F} 1$ score of $88.2 \%$. It outperforms the typical $\mathrm{GAC}$, and the close-to-target initial contour helps to achieve a faster segment of $\mathrm{CC}$.

\section{REFERENCES}

[1] P. Conlon and M.R. Trimble, "A Study of the Corpus Callosum in Epilepsy using Magnetic Resonance Imaging”, Epilepsy Research, Vol. 2, No. 2, pp. 122-126, 1988.

[2] H. Chahboune et al., "DTI Abnormalities in Anterior Corpus Callosum of Rats with Spike-Wave Epilepsy," Neuroimage, Vol. 47, No. 2, pp. 459-466, 2009.

[3] Q. He, Y. Duan, J. Miles and N. Takahashi, "Statistical Shape Analysis of the Corpus Callosum in Subtypes of Autism", Proceedings of $7^{\text {th }}$ Annual International Conference on Bio Engineering, pp. 1087-1091, 2007.

[4] Q. He, K. Karsch and Y. Duan, "Abnormalities in MRI Traits of Corpus Callosum in Autism Subtype", Proceedings of $30^{\text {th }}$ Annual International Conference of the IEEE 
Engineering in Medicine and Biology Society, pp. 39003903, 2008.

[5] K. Von Plessen, A. Lundervold, N. Duta, E. Heiervang, F. Klauschen, A. Smievoll, L. Ersland and K. Hugdahl, "Less Developed Corpus Callosum in Dysplexic Subjects-A Structural MRI Study", Neuropsychologia, Vol. 40, No. 7, pp. 1035-1044, 2002.

[6] J.G. Fine, M. Semrud-Clikeman, T.Z. Keith, L.M. Stapleton and G.W. Hynd, "Reading and the Corpus Callosum: An MRI Family Study of Volume and Area", Neuropsychology, Vol. 21, No. 2, pp. 235-241, 2007.

[7] Medical Imaging, Available at: http://www.cihi.ca/CIHIext-

portal/internet/EN/TabbedContent/types+of+care/specialize d+services/medical+imaging/cihi010642.

[8] H.T. Chugani, "Neuroimaging in Epilepsy", Oxford University Press, 2011.

[9] M. Brejl and M. Sonka, "Object Localization and Border Detection Criteria Design in Edge-based Image Segmentation: Automated Learning from Examples", IEEE Transactions on Medical Imaging, Vol. 19, No. 10, pp. 973985, 2000.

[10] B.V. Ginneken, A.F. Frangi, J.J. Staal, B.M.T.H. Romeny and M.A. Viergever, "Active Shape Model Segmentation with Optimal Features", IEEE Transactions on Medical Imaging, Vol. 21, No. 8, pp. 924-933, 2002.

[11] M. Jacob, T. Blu and M. Unser, "Efficient Energies and Algorithms for Parametric Snakes", IEEE Transactions on Image Processing, Vol. 13, No. 9, pp. 1231-1244, 2004.

[12] R. Sandhu, T. Georgiou, and A. Tannenbaum, "A New Distribution Metric for Image Segmentation", Technical Paper, Georgia Institute of Technology, pp. 1-9, 2008.

[13] J. Zhou, S. Chang, S. Zhang, G. Pappas, M. Michaelides, F. Delis, N. Volkow, P. Thanos and D. Metaxas, "A Novel Learning based Segmentation Method for Rodent Brain Structures using MRI", Proceedings of $5^{\text {th }}$ IEEE International Symposium on Biomedical Imaging: From Nano to Macro, pp. 61-64, 2008.
[14] N. El-Zehiry, M. Casanova and A. Elmaghraby, "Variability of the Relative Corpus Callosum Cross Sectional Area between Dyslexic and Normally Developed Brains", Proceedings of $5^{\text {th }}$ IEEE International Symposium on Biomedical Imaging: From Nano to Macro, pp. 436-439, 2008.

[15] Rongjie Lai, Yonggang Shi, Nancy Sicotte and Arthur W. Toga, "Automated Corpus Callosum Extraction via LaplaceBeltrami Nodal Parcellation and Intrinsic Geodesic Curvature Flows on Surfaces", Proceedings of IEEE International Conference on Computer Vision, pp. 20342040, 2011.

[16] A. Mayer and H. Greenspan, "An Adaptive Mean-Shift Framework for MRI Brain Segmentation", IEEE Transactions on Medical Imaging, Vol. 28, No. 8, pp. 12381250, 2009.

[17] Tao $\mathrm{Xu}$, Irene Cheng and Mrinal Mandal, "Automated Cavity Detection of Infectious Pulmonary Tuberculosis in Chest Radiographs", Proceedings of Annual International Conference of the IEEE Engineering in Medicine and Biology Society, pp. 5178-5181, 2011.

[18] Daniel S. Marcus, Tracy H. Wang, Jamie Parker and John G. Csernansky, "Open Access Series of Imaging Studies (OASIS):Cross-Sectional MRI Data in Young, Middle Aged, Nondemented, and Demented Older Adults", Journal of Cognitive Neuroscience, Vol. 19, No. 9, pp. 1498-1507, 2007.

[19] T. Xu, M. Mandal, R. Long, I. Cheng and A. Basu, "An Edge-Region Force Guided Active Shape Approach for Automatic Lung Field Detection in Chest Radiographs", Computerized Medical Imaging and Graphics, Vol. 36, No. 6, pp. 452-63, 2012.

[20] S. Anand, S. Mittal, O. Tuzel and P. Meer, "SemiSupervised Kernel Mean Shift Clustering", IEEE Transactions on Pattern Analysis and Machine Intelligence, Vol. 36, No. 6, pp. 1201-1215, 2014. 